\title{
Vector Boson Fusion Production of the Standard Model Higgs at the LHC
}

\author{
Mónica Luisa Vázquez Acosta (on behalf of the CMS Collaboration)* \\ Imperial College London \\ E-mail: monicava@mail.cern.ch
}

\begin{abstract}
The cross section measurements of the Higgs boson production in the vector boson fusion (VBF) process at the LHC followed by a Higgs boson decay into $\tau \tau, W W$ and $\gamma \gamma$ will significantly extend the possibility of Higgs boson coupling measurements. Prospective analyses with the CMS experiment are discussed for the $H \rightarrow \gamma \gamma, W W$ and $\tau \tau$ decay channels for an integrated LHC luminosity of $30 \mathrm{fb}^{-1}$. For a Higgs boson mass in the range 115 to $140 \mathrm{GeV}$, an observation with a significance above 2 standard deviations is expected in the $\mathrm{H}$ to $\gamma \gamma$ channel, and above 3 standard deviations in the $\mathrm{H}$ to $\tau \tau$ channel. The $\mathrm{H}$ to WW channel offers a discovery reach above 5 sigma in the mass range 140 to $200 \mathrm{GeV}$. A new complete strategy is presented for the control of systematics and early searches at very low luminosities of the order of $1 \mathrm{fb}^{-1}$.
\end{abstract}

2008 Physics at LHC

September 29 - 4 October 2008

Split, Croatia

\footnotetext{
* Speaker.
} 


\section{Introduction}

Vector Boson Fusion (VBF) Higgs boson production is the second largest production mechanism at the LHC. The cross section measurements of the VBF process, $V V \rightarrow H(q q \rightarrow q q H)$, followed by Higgs boson decays into $\tau \tau, W W$ and $\gamma \gamma$ will significantly extend the possibility of Higgs boson coupling measurements [1,2].

\section{Vector Boson Fusion Signature}

Events produced by VBF are characterized by a distict topology of the final state: two forward jets with little extra hadronic activity and the decay products of the Higgs boson. The rapidity distribution of the 3rd jet with respect to the two forward jets, $\eta_{\mathrm{j} 3}^{*}$, is shown in Fig. 1 (left) which shows a double-peak structure for the electroweak processes, including the VBF signal, and is more central for the QCD background samples. Applying a central jet veto (CJV) is a poweful rejection method against the QCD background. To avoid considering jets from pile-up events in the CJV, jets are associated to the signal vertex using tracks. For every extra jet one can define the quantity $\alpha_{\mathrm{j} 3}=\Sigma \mathrm{p}_{\mathrm{Ttrk}} / \mathrm{E}_{\mathrm{Tj} 3}$, where $\mathrm{p}_{\mathrm{Ttrk}}$ is the $\mathrm{p}_{\mathrm{T}}$ of tracks from the signal vertex within the jet cone and $\mathrm{E}_{\mathrm{Tj} 3}$ is the jet measured raw $\mathrm{E}_{\mathrm{T}}$. Figure 1 (right) shows $\alpha_{3 \mathrm{j}}$ tends to peak at low values for non-signal jets. The efficiency of the veto for the background samples versus the signal efficiency is shown in Figure 2 (left) for events containg a 3rd jet with $E_{T}$ larger than different threshold values. An optimal threshold where the signal process has $\sim 80 \%$ efficiency while the backgrounds are suppressed below 50\% is used [3]. An alternative approach is to consider a track counting veto (TCV) [4], where the number of tracks between the two leading jets is counted with different $p_{T}$ thresholds. Figure 2 (right) shows the performance of the TCV algorithm, i.e the efficiency of selecting the signal versus the background for events with an increasing cut on the track multiplicity and $p_{T}$. The black star indicates the performance of the CJV based on calorimeter jets. The TCV algorithm can reach similar discrimination power than the central jet veto.
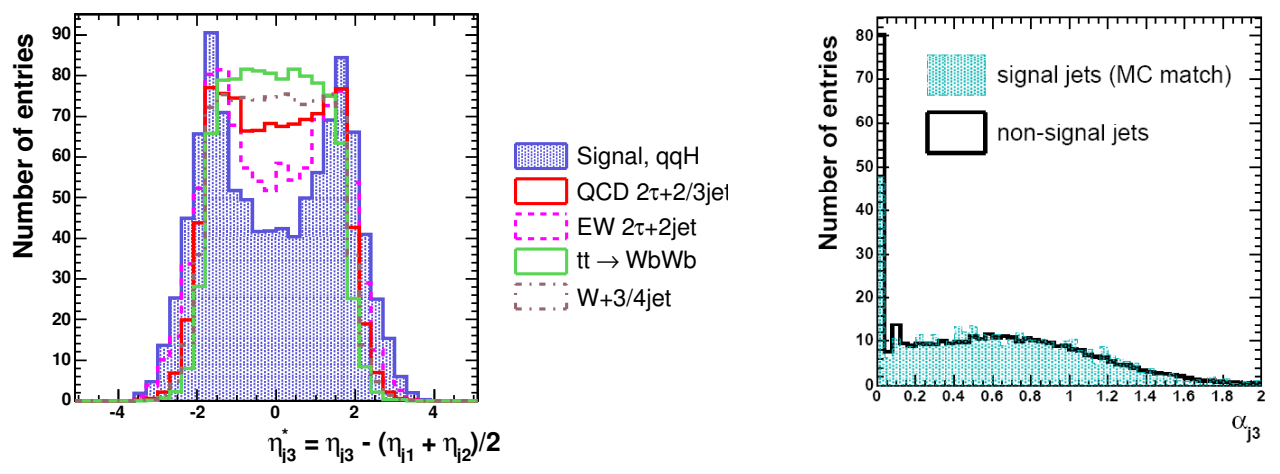

Figure 1: The $\eta$ distribution of the 3rd jet with respect to the two forward jets (left). The distribution of $\alpha_{3 j}$ which is used to match jets to the signal vertex (right). 

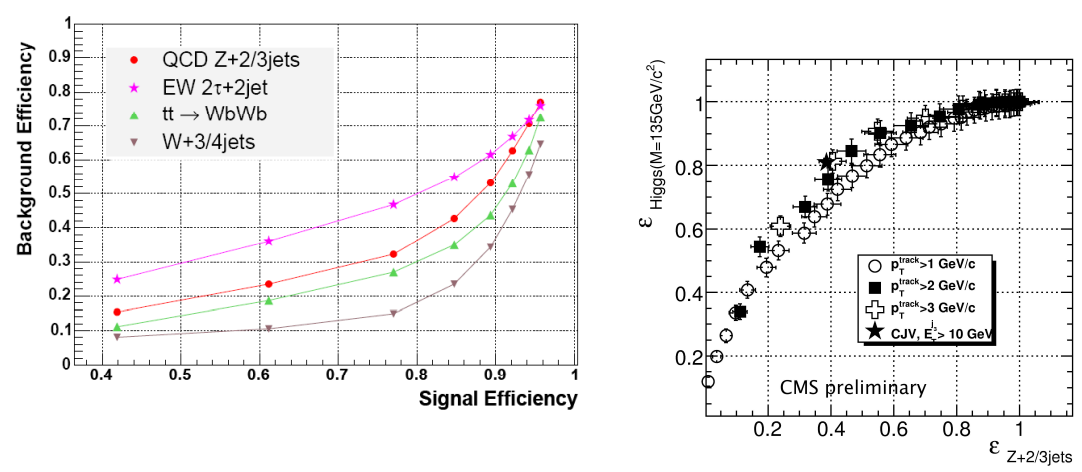

Figure 2: Efficiency of the CJV for background versus signal $\left(\mathrm{M}_{\mathrm{H}}=135 \mathrm{GeV}\right)$, for increasing 3rd jet $\mathrm{E}_{\mathrm{Tth}}$ threshold (left). TCV performance for different $p_{t}^{\text {track }}$ and track multiplicity thresholds compared to the performance of the CJV.

\section{Vector Boson Fusion Higgs Discovery Potential}

The observability of the VBF Higgs boson production has been studied with the full CMS detector simulation in the $H \rightarrow \tau \tau, \gamma \gamma$ and $W W$ decay channels [5]. VBF $H \rightarrow \tau \tau$ production has been studied in the Higgs mass range of 115 to $145 \mathrm{GeV}$ in the lepton plus $\tau_{\text {jet }}$ final state. Figure 3 (left) shows the expected di- $\tau$ mass distribution using the collinear approximation [3] for a luminosity of $30 \mathrm{fb}^{-1}$. Figure 3 (right) shows the significance of the expected number of signal events for different Higgs masses. A statistical signal significance of $3.9 \sigma$ is expected for a Higgs mass of $135 \mathrm{GeV}$.

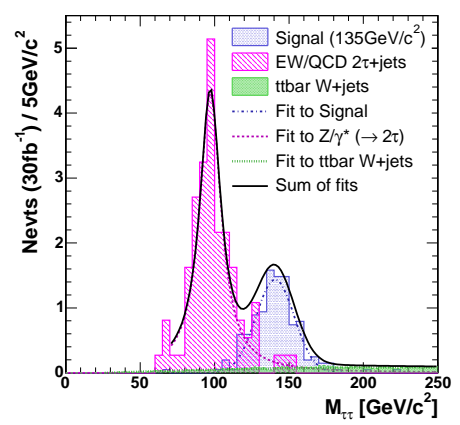

\begin{tabular}{|l||c|c|c|c|}
\hline $\mathrm{M}_{H}[\mathrm{GeV}]$ & 115 & 125 & 135 & 145 \\
\hline \hline $\mathrm{N}_{\mathrm{S}}\left(30 \mathrm{fb}^{-1}\right)$ & 10.47 & 7.79 & 7.94 & 3.63 \\
\hline $\mathrm{N}_{\mathrm{B}}\left(30 \mathrm{fb}^{-1}\right)$ & 3.70 & 2.21 & 1.84 & 1.42 \\
\hline $\mathrm{S}_{C P}$ at $30 \mathrm{fb}^{-1}($ no uncertainty) & 4.04 & 3.71 & 3.98 & 2.19 \\
$\mathrm{~S}_{c P}$ at $30 \mathrm{fb}^{-1}\left(\sigma_{B}=7.8 \%\right)$ & 3.97 & 3.67 & 3.94 & 2.18 \\
\hline $\mathrm{S}_{C P}$ at $60 \mathrm{fb}^{-1}\left(\sigma_{B}=5.9 \%\right)$ & 5.67 & 5.26 & 5.64 & 3.19 \\
\hline
\end{tabular}

Figure 3: Di- $\tau$ invariant mass expected for a luminosity of $30 \mathrm{fb}^{-1}$ (left). Significance of the expected number of signal events for different Higgs boson masses (right).

VBF $H \rightarrow W W$ production in the lepton plus two jet final state has been studied in the Higgs mass range between 120 and $250 \mathrm{GeV}$. Figure 4 (left) shows the signal significance expected with $30 \mathrm{fb}^{-1}$ for different central jet veto selections [6]. In the mass range between 140-200 GeV a $5 \sigma$ significance can be achieved. VBF $H \rightarrow \gamma \gamma$ production has also been studied in the Higgs mass range between 115 and $150 \mathrm{GeV}$ [7]. Figure 4 (right) shows the signal significance expected with 30 and $60 \mathrm{fb}^{-1}$. With $60 \mathrm{fb}^{-1}$ of collected data a $3 \sigma$ significance can be achieved for a low mass Higgs in the range 115 to $130 \mathrm{GeV}$. 

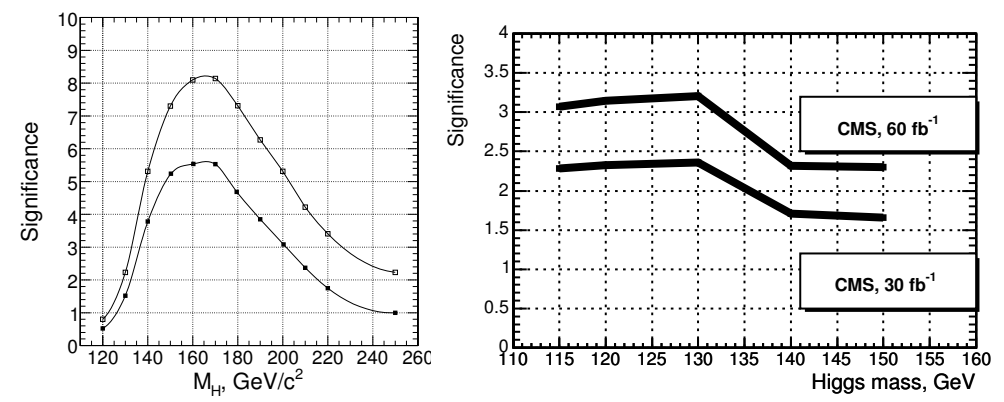

Figure 4: Signal significance of VBF $H \rightarrow W W$ for $30 \mathrm{fb}^{-1}$. The high (low) curves correspond to full (loose) extra jet veto (left). Signal significance of VBF $H \rightarrow \gamma \gamma$ for 30 and $60 \mathrm{fb}^{-1}$ (right).

\section{Search of Higgs $\rightarrow \tau \tau \rightarrow$ lepton $+\tau_{\text {jet }}$ with $\mathbf{1} \mathbf{f b}^{-1}$}

A selection strategy for the search of VBF Higgs $\rightarrow \tau \tau \rightarrow$ lepton $+\tau_{\text {jet }}$ with $1 \mathrm{fb}^{-1}$ has been developed and is described in detail in [8]. The di- $\tau$ invariant mass will be analyzed to search for the presence of a Higgs boson in the region above the $Z \rightarrow \tau \tau$ mass peak. It is important to know well the shape of the $Z \rightarrow \tau \tau$ background. The dominant uncertainty comes from the modeling of the missing transverse momentum related to the effects of pile-up, underlying event and the calorimeter noise and response. A method to model the di- $\tau$ mass has been developed [4]. $Z \rightarrow \mu \mu$ data events are selected and the muons are removed from the real event. Di- $\tau$ Monte Carlo events are generated with the same kinematics as the real muons and their detector response is fully simulated. Finally the real $Z \rightarrow \mu \mu$ events with the muons removed and the simulated di- $\tau$ events are super-imposed to form one event, $Z \rightarrow \tau_{\mu} \tau_{\mu}$, and the di- $\tau$ mass is calculated. The reconstructed di- $\tau$ mass for real and fake $Z \rightarrow \tau \tau$ events for inclusive Drell-Yan and $Z+$ jets events are shown in Fig. 5. A good agreement between the di- $\tau$ mass shapes is obtained.

The expected di- $\tau$ mass distribution for the background and the Higgs signal for $1 \mathrm{fb}^{-1}$ is shown in Fig. 6 (left). A profile likelihood method is used to evaluate the upper limit on the number of signal events. Figure 6 (right) shows the expected 95\% CL limit on the cross section times branching ratio as a function of the Higgs boson mass.
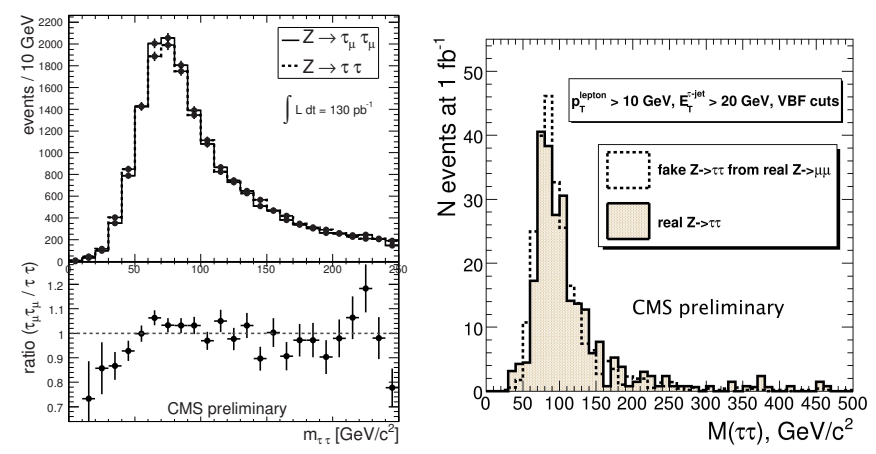

Figure 5: Reconstructed di- $\tau$ mass for real and fake $Z \rightarrow \tau \tau$ events for the final states (left) $\tau \tau \rightarrow \mu \nu \nu+\mu \nu v$ from inclusive Drell-Yan events and (right) $\tau \tau \rightarrow l v v+\tau_{\text {jet }} v$ from $Z+$ jets events. 

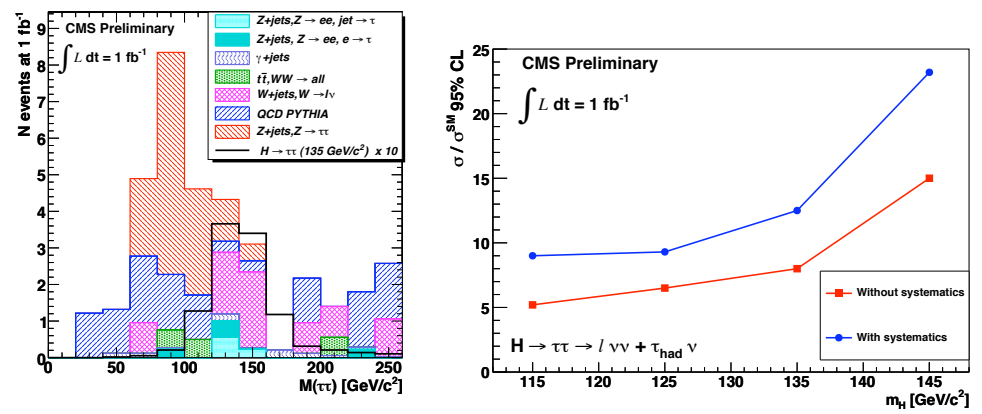

Figure 6: Di- $\tau$ mass distribution of expected backgrounds with $1 \mathrm{fb}^{-1}$ after all selection. Backgrounds are shown cumulative. The signal mass distribution scaled by a factor 10 is also shown for $M_{H}=135 \mathrm{GeV}$.

\section{Conclusion}

A selection strategy for the Standard Model Higgs boson produced in vector boson fusion decaying to a pair of $\tau$ leptons with $1 \mathrm{fb}^{-1}$ of early CMS data at the LHC has been presented. No signal evidence is expected and upper limit on the cross section times branching ratio is evaluated. Prospective analyses for the $H \rightarrow \gamma \gamma, W W$ and $\tau \tau$ decay channels for a luminosity of $30 \mathrm{fb}^{-1}$ have also been discussed. For a Higgs boson mass in the range 115 to $140 \mathrm{GeV}$, an observation with a significance above 2 standard deviations is expected in the $\mathrm{H}$ to $\gamma \gamma$ channel, and above 3 standard deviations in the $\mathrm{H}$ to $\tau \tau$ channel. The $\mathrm{H}$ to WW channel offers a discovery reach above 5 sigma in the mass range of 140 to $200 \mathrm{GeV}$.

\section{References}

[1] D. Zeppenfeld et al., Measuring Higgs boson couplings at the LHC, Phys. Rev. D62 (2000) 013009

[2] M. Duhrssen, M. et al., Extracting Higgs boson couplings from LHC data, Phys. Rev. D70 (2004) 113009

[3] C. Foudas, A. Nikitenko, M. Takahashi, Observation of the Standard Model Higgs boson via $H \rightarrow \tau \tau \rightarrow$ lepton + jet Channel, CMS Note 2006/088 (2006)

[4] CMS Collaboration, Towards the Search for the Standard Model Higgs boson produced in Vector Boson Fusion and decaying into a $\tau$ pair in CMS with $1 \mathrm{fb}^{-1}: \tau$ identification studies, CMS PAS HIG-08-001 (2008)

[5] CMS Collaboration, CMS Physics Technical Design Report Volume II: Physics Performance, CERN/LHCC 2006-021 CMS TDR 8.2 (2006)

[6] H. Pi et al., Search for Standard Model Higgs Boson via Vector Boson Fusion in the $H \rightarrow W^{+} W^{-} \rightarrow l^{ \pm} v j j$ with $120<\mathrm{m}_{\mathrm{H}}<250 \mathrm{GeV} / \mathrm{c}^{2}, C M S$ Note 2006/092 (2006)

[7] M. Dubinin et al., Vector Boson Fusion Production with $H \rightarrow \gamma \gamma, C M S$ Note 2006/097 (2006)

[8] CMS Collaboration, Search for the Standard Model Higgs boson produced in Vector Boson Fusion and decaying into a $\tau$ pair in CMS with $1 \mathrm{fb}^{-1}$, CMS PAS HIG-08-008 (2008) 